Supplement of SOIL, 5, 91-105, 2019

https://doi.org/10.5194/soil-5-91-2019-supplement

(C) Author(s) 2019. This work is distributed under

the Creative Commons Attribution 4.0 License.

(c) (1)

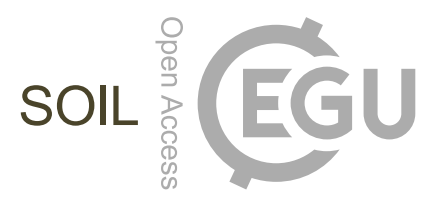

Supplement of

\title{
On-farm study reveals positive relationship between gas transport capac- ity and organic carbon content in arable soil
}

Tino Colombi et al.

Correspondence to: Tino Colombi (tino.colombi@slu.se)

The copyright of individual parts of the supplement might differ from the CC BY 4.0 License. 
Supplemental Table S1: Summary statistics of multiple linear regression models (Eq. 3) to explain soil organic carbon content as a function of gas diffusivity and clay content in the management systems with and without regular tillage. ** and * indicate significant regression coefficients at $p<0.01$ and $p<0.05$, respectively, ns indicates nonsignificant regression coefficients. $R^{2}$ represents multiple r-squared.

\begin{tabular}{ccccccc}
\hline Management & $\boldsymbol{\Psi}[\mathbf{h P a}]$ & Depth [cm] & Dp/D0 [-] & Clay [\%] & Int & $\mathbf{R}^{2}$ \\
\hline CON \& ORG & 30 & $-12.5 \mathrm{~cm}$ & $434.0^{*}$ & $1.001^{* *}$ & $-10.94 \mathrm{~ns}$ & 0.71 \\
& \multirow{2}{*}{100} & $-37.5 \mathrm{~cm}$ & $756.5 \mathrm{~ns}$ & $0.758^{* *}$ & $-11.68 \mathrm{~ns}$ & 0.52 \\
& \multirow{2}{*}{30} & $-37.5 \mathrm{~cm}$ & $349.5 \mathrm{~ns}$ & $0.728^{* *}$ & $-9.766 \mathrm{~ns}$ & 0.50 \\
NT & $-12.5 \mathrm{~cm}$ & $744.0^{*}$ & $0.954^{*}$ & $-10.79 \mathrm{~ns}$ & 0.66 \\
& \multirow{2}{*}{100} & $-37.5 \mathrm{~cm}$ & $318.2 \mathrm{~ns}$ & $0.538^{*}$ & $-5.165 \mathrm{~ns}$ & 0.48 \\
& $-12.5 \mathrm{~cm}$ & $420.8^{*}$ & $0.996^{*}$ & $-12.34 \mathrm{~ns}$ & 0.68 \\
& $-37.5 \mathrm{~cm}$ & $349.7 \mathrm{~ns}$ & $0.606^{*}$ & $-8.421 \mathrm{~ns}$ & 0.63 \\
\hline
\end{tabular}

Abbreviations: CON is conventional, ORG is organic, NT is no-till, $\Psi$ is soil matric suction, Dp/D0 is gas diffusion coefficient, Clay is clay content, Int is intercept

Supplemental Table S2: Summary statistics of multiple linear regression models (Eq. 3) to explain soil organic carbon content as a function of air permeability and clay content in the management systems with and without regular tillage. $* *$ and $*$ indicate significant regression coefficients at $p<0.01$ and $p<0.05$, respectively, ns indicates nonsignificant regression coefficients. $R^{2}$ represents multiple r-squared.

\begin{tabular}{ccccccc}
\hline Management & $\boldsymbol{\Psi}[\mathbf{h P a}]$ & Depth $[\mathbf{c m}]$ & $\mathbf{K a}\left[\boldsymbol{\mu m ^ { 2 }}\right]$ & Clay $[\%]$ & Int & $\mathbf{R}^{\mathbf{2}}$ \\
\hline CON \& ORG & 30 & $-12.5 \mathrm{~cm}$ & $0.159 \mathrm{~ns}$ & $0.965^{* *}$ & $-7.740 \mathrm{~ns}$ & 0.65 \\
& \multirow{2}{*}{100} & $-37.5 \mathrm{~cm}$ & $0.263^{* *}$ & $0.664^{* *}$ & $-7.464 \mathrm{~ns}$ & 0.70 \\
& & $-12.5 \mathrm{~cm}$ & $0.095^{*}$ & $1.054^{* *}$ & $-12.48 \mathrm{~ns}$ & 0.68 \\
\multirow{2}{*}{ NT } & \multirow{2}{*}{30} & $-37.5 \mathrm{~cm}$ & $0.160^{* *}$ & $0.707^{* *}$ & $-8.363 \mathrm{~ns}$ & 0.62 \\
& \multirow{2}{*}{100} & $-37.5 \mathrm{~cm}$ & $0.284^{*}$ & $0.529^{* *}$ & $-5.196 \mathrm{~ns}$ & 0.67 \\
& & $-12.5 \mathrm{~cm}$ & $0.146^{*}$ & $1.004^{* *}$ & $-9.671 \mathrm{~ns}$ & 0.55 \\
& & $-37.5 \mathrm{~cm}$ & $0.160^{*}$ & $0.543^{* *}$ & $-5.732 \mathrm{~ns}$ & 0.70
\end{tabular}

Abbreviations: CON is conventional, ORG is organic, NT is no-till, $\Psi$ is soil matric suction, Ka is air permeability, Clay is clay content, Int is intercept 
Supplemental Table S3: Summary statistics of multiple linear regression models (Eq. 3) to explain soil organic carbon content as a function of air-filled porosity and clay content in the management systems with and without regular tillage. ** and * indicate significant regression coefficients at $p<0.01$ and $p<0.05$, respectively, $n$ indicates nonsignificant regression coefficients. $R^{2}$ represents multiple r-squared.

\begin{tabular}{ccccccc}
\hline Management & $\boldsymbol{\Psi}[\mathbf{h P a}]$ & Depth $[\mathbf{c m}]$ & $\mathbf{\varepsilon a}\left[\mathbf{c m}^{\mathbf{3}} \mathbf{c m}^{-3}\right]$ & Clay [\%] & Int & $\mathbf{R}^{\mathbf{2}}$ \\
\hline CON \& ORG & 30 & $-12.5 \mathrm{~cm}$ & $0.730 \mathrm{~ns}$ & $0.951^{* *}$ & $-14.02 \mathrm{~ns}$ & 0.60 \\
& & $-37.5 \mathrm{~cm}$ & $0.638 \mathrm{~ns}$ & $0.788^{* *}$ & $-13.30 \mathrm{~ns}$ & 0.46 \\
& 100 & $-12.5 \mathrm{~cm}$ & $1.110 \mathrm{~ns}$ & $0.979^{* *}$ & $-24.61 \mathrm{~ns}$ & 0.65 \\
NT & $-37.5 \mathrm{~cm}$ & $0.630 \mathrm{~ns}$ & $0.815^{* *}$ & $-15.05 \mathrm{~ns}$ & 0.46 \\
& \multirow{2}{*}{30} & $-12.5 \mathrm{~cm}$ & $2.164 \mathrm{~ns}$ & $0.742 \mathrm{~ns}$ & $-23.53 \mathrm{~ns}$ & 0.46 \\
& \multirow{2}{*}{100} & $-37.5 \mathrm{~cm}$ & $0.759^{*}$ & $0.431^{*}$ & $-9.209 \mathrm{~ns}$ & 0.67 \\
& $-12.5 \mathrm{~cm}$ & $2.253 \mathrm{~ns}$ & $0.992^{*}$ & $-35.69 \mathrm{~ns}$ & 0.51 \\
& & $-37.5 \mathrm{~cm}$ & $0.737^{*}$ & $0.418^{*}$ & $-9.974 \mathrm{~ns}$ & 0.70
\end{tabular}

Abbreviations: CON is conventional, ORG is organic, NT is no-till, $\Psi$ is soil matric suction, $\varepsilon$ a is air-filled porosity, Clay is clay content, Int is intercept

Supplemental Table S4: Summary statistics of multiple linear regression models (Eq. 3) to explain soil organic carbon content as a function of water holding capacity and clay content in the management systems with and without regular tillage. ** and * indicate significant regression coefficients at $p<0.01$ and $p<0.05$, respectively, ns indicates nonsignificant regression coefficients. $R^{2}$ represents multiple r-squared.

\begin{tabular}{cccccc}
\hline Management & Depth [cm] & WHC $\left[\mathbf{g}^{\mathbf{1}} \mathbf{g}^{-1}\right]$ & Clay [\%] & Int & $\mathbf{R}^{\mathbf{2}}$ \\
\hline CON \& ORG & $-12.5 \mathrm{~cm}$ & $108.2^{* *}$ & $0.176 \mathrm{~ns}$ & $-16.98^{* *}$ & 0.77 \\
& $-37.5 \mathrm{~cm}$ & $97.16^{*}$ & $-0.022 \mathrm{~ns}$ & $-9.018 \mathrm{~ns}$ & 0.57 \\
NT & $-12.5 \mathrm{~cm}$ & $123.2 \mathrm{~ns}$ & $-0.122 \mathrm{~ns}$ & $-10.83 \mathrm{~ns}$ & 0.28 \\
& $-37.5 \mathrm{~cm}$ & $21.33 \mathrm{~ns}$ & $0.345 \mathrm{~ns}$ & $-2.640 \mathrm{~ns}$ & 0.42 \\
\hline
\end{tabular}

Abbreviations: CON is conventional, ORG is organic, NT is no-till, WHC is water holding capcity, Clay is clay content, Int is intercept 
Supplemental Table S5: Summary statistics of multiple linear regression models (Eq. 3) to explain soil organic carbon content as a function of gas diffusivity, clay content and sampling time. $* *$ and $*$ indicate significant regression coefficients at $\mathbf{p}<0.01$ and $\mathbf{p}<$ 0.05 , respectively, $n$ indicates nonsignificant regression coefficients. $R^{2}$ represents multiple r-squared.

\begin{tabular}{ccccccc}
\hline $\boldsymbol{\Psi}[\mathbf{h P a}]$ & Depth [cm] & Dp/D0 [-] & Clay [\%] & Date & Int & $\mathbf{R}^{2}$ \\
\hline 30 & $-12.5 \mathrm{~cm}$ & $424.3^{* *}$ & $0.933^{* *}$ & $-0.080 \mathrm{~ns}$ & $4.304 \mathrm{~ns}$ & 0.67 \\
& $-37.5 \mathrm{~cm}$ & $592.9 \mathrm{~ns}$ & $0.744^{* *}$ & $-0.062 \mathrm{~ns}$ & $-0.944 \mathrm{~ns}$ & 0.54 \\
\multirow{2}{*}{100} & $-12.5 \mathrm{~cm}$ & $256.9^{* *}$ & $0.956^{* *}$ & $-0.070 \mathrm{~ns}$ & $0.802 \mathrm{~ns}$ & 0.69 \\
& $-37.5 \mathrm{~cm}$ & $339.5^{*}$ & $0.717^{* *}$ & $-0.083 \mathrm{~ns}$ & $3.132 \mathrm{~ns}$ & 0.55
\end{tabular}

Abbreviations: $\Psi$ is soil matric suction, Dp/D0 is gas diffusion coefficient, Clay is clay content, Date is day of the year when samples were taken, Int is intercept

5 Supplemental Table S6: Summary statistics of multiple linear regression models (Eq. 3) to explain soil organic carbon content as a function of air permeability, clay content and sampling time. ** and * indicate significant regression coefficients at $p<0.01$ and $p<$ 0.05 , respectively, $n s$ indicates nonsignificant regression coefficients. $R^{2}$ represents multiple r-squared.

\begin{tabular}{ccccccc}
\hline $\boldsymbol{\Psi}[\mathbf{h P a}]$ & Depth $[\mathbf{c m}]$ & $\mathbf{K a}\left[\boldsymbol{\mu m}^{2}\right]$ & Clay [\%] & Date & Int & $\mathbf{R}^{2}$ \\
\hline 30 & $-12.5 \mathrm{~cm}$ & $0.145^{*}$ & $0.879^{* *}$ & $-0.101 \mathrm{~ns}$ & $12.23 \mathrm{~ns}$ & 0.62 \\
& $-37.5 \mathrm{~cm}$ & $0.293^{* *}$ & $0.658^{* *}$ & $-0.102 \mathrm{~ns}$ & $-5.776 \mathrm{~ns}$ & 0.71 \\
\multirow{2}{*}{100} & $-12.5 \mathrm{~cm}$ & $0.075^{*}$ & $0.947^{* *}$ & $-0.077 \mathrm{~ns}$ & $4.799 \mathrm{~ns}$ & 0.62 \\
& $-37.5 \mathrm{~cm}$ & $0.158^{* *}$ & $0.689^{* *}$ & $-0.039 \mathrm{~ns}$ & $-2.178 \mathrm{~ns}$ & 0.65 \\
\hline
\end{tabular}

Abbreviations: $\Psi$ is soil matric suction, Ka is air permeability, Clay is clay content, Date is day of the year when samples were taken, Int is intercept

Supplemental Table S7: Summary statistics of multiple linear regression models (Eq. 3) to explain soil organic carbon content as a 10 function of air-filled porosity, clay content and sampling time. ** indicates significant regression coefficients at $\mathbf{p}<0.01$, ns indicates nonsignificant regression coefficients. $R^{2}$ represents multiple r-squared.

\begin{tabular}{ccccccc}
\hline $\boldsymbol{\Psi}[\mathbf{h P a}]$ & Depth $[\mathbf{c m}]$ & $\mathbf{8 a}\left[\mathbf{c m}^{\mathbf{3}} \mathbf{c m}^{-\mathbf{3}}\right]$ & Clay $[\%]$ & Date & Int & $\mathbf{R}^{\mathbf{2}}$ \\
\hline 30 & $-12.5 \mathrm{~cm}$ & $0.501 \mathrm{~ns}$ & $0.837^{* *}$ & $-0.168 \mathrm{~ns}$ & $19.39 \mathrm{~ns}$ & 0.56 \\
& $-37.5 \mathrm{~cm}$ & $0.510 \mathrm{~ns}$ & $0.728^{* *}$ & $-0.114 \mathrm{~ns}$ & $6.698 \mathrm{~ns}$ & 0.50 \\
\multirow{2}{*}{100} & $-12.5 \mathrm{~cm}$ & $0.639 \mathrm{~ns}$ & $0.857^{* *}$ & $-0.163 \mathrm{~ns}$ & $14.29 \mathrm{~ns}$ & 0.59 \\
& $-37.5 \mathrm{~cm}$ & $0.506 \mathrm{~ns}$ & $0.746^{* *}$ & $-0.115 \mathrm{~ns}$ & $5.476 \mathrm{~ns}$ & 0.50
\end{tabular}

Abbreviations: $\Psi$ is soil matric suction, $\varepsilon$ a is air-filled porosity, Clay is clay content, Date is day of the year when samples were taken, Int is intercept 
Supplemental Table S8: Summary statistics of multiple linear regression models (Eq. 3) to explain soil organic carbon content as a function of water holding capacity, clay content and sampling time. ** indicates significant regression coefficients at $\mathbf{p}<0.01$, ns indicates nonsignificant regression coefficients. $R^{2}$ represents multiple r-squared.

\begin{tabular}{cccccc}
\hline Depth $[\mathbf{c m}]$ & WHC $\left[\mathbf{g ~ g}^{-1}\right]$ & Clay [\%] & Date & Int & $\mathbf{R}^{2}$ \\
\hline$-12.5 \mathrm{~cm}$ & $85.72^{* *}$ & $0.249 \mathrm{~ns}$ & $-0.129 \mathrm{~ns}$ & $9.380 \mathrm{~ns}$ & 0.66 \\
$-37.5 \mathrm{~cm}$ & $84.98^{* *}$ & $0.091 \mathrm{~ns}$ & $-0.137 \mathrm{~ns}$ & $12.18 \mathrm{~ns}$ & 0.62 \\
\hline
\end{tabular}

Abbreviations: WHC is water holding capacity, Clay is clay content, Date is day of the year when samples were taken, Int is intercept

5 Supplemental Table S9: Summary statistics of multiple linear regression models (Eq. 3) to explain soil organic carbon content as a function of clay content and exogenous organic matter input estimated as described by Büchi et al. (2019). ** indicates significant regression coefficients at $\mathbf{p}<0.01$, ns indicates nonsignificant regression coefficients. $R^{2}$ represents multiple $r$-squared.

\begin{tabular}{ccccc}
\hline Depth [cm] & Clay [\%] & OC Inp $\left[\mathbf{k g ~ C ~ h a ~}^{-\mathbf{1}} \mathbf{y}^{-1}\right]$ & Int & $\mathbf{R}^{\mathbf{2}}$ \\
\hline$-12.5 \mathrm{~cm}$ & $0.831^{* *}$ & $0.008 \mathrm{~ns}$ & $-4.520 \mathrm{~ns}$ & 0.52 \\
$-37.5 \mathrm{~cm}$ & $0.636^{* *}$ & $0.003 \mathrm{~ns}$ & $-5.267 \mathrm{~ns}$ & 0.44 \\
\hline
\end{tabular}

Abbreviations: Clay is clay content, OC Inp is Exogenous organic carbon input, Int is intercept

Supplemental Table S10: Summary statistics of multiple linear regression models (Eq. 3) to explain soil organic carbon content as a 10 function gas diffusivity, clay content and exogenous organic matter input estimated as described by Büchi et al. (2019). ** and * indicate significant regression coefficients at $p<0.01$ and $p<0.05$, respectively, ns indicates nonsignificant regression coefficients. $\mathbf{R}^{2}$ represents multiple r-squared.

\begin{tabular}{ccccccc}
\hline $\boldsymbol{\Psi}[\mathbf{h P a}]$ & Depth [cm] & Dp/D0 [-] & Clay [\%] & OC Inp [kg C ha $\left.\mathbf{~ y}^{-1}\right]$ & Int & $\mathbf{R}^{\mathbf{2}}$ \\
\hline 30 & $-12.5 \mathrm{~cm}$ & $429.2^{* *}$ & $0.927^{* *}$ & $0.003 \mathrm{~ns}$ & $-9.957^{*}$ & 0.67 \\
& $-37.5 \mathrm{~cm}$ & $853.3^{* *}$ & $0.776^{* *}$ & $0.001 \mathrm{~ns}$ & $-13.81^{* *}$ & 0.58 \\
\multirow{2}{*}{100} & $-12.5 \mathrm{~cm}$ & $258.0^{* *}$ & $0.946^{* *}$ & $0.003 \mathrm{~ns}$ & $-12.05^{*}$ & 0.69 \\
& $-37.5 \mathrm{~cm}$ & $487.9^{* *}$ & $0.740^{* *}$ & $0.003 \mathrm{~ns}$ & $-1.218^{*}$ & 0.58 \\
\hline
\end{tabular}

Abbreviations: $\Psi$ is soil matric suction, Dp/D0 is gas diffusion coefficient, Clay is clay content, OC Inp is Exogenous organic carbon input, Int is intercept 
Supplemental Table S11: Summary statistics of multiple linear regression models (Eq. 3) to explain soil organic carbon content as a function of air permeability, clay content and exogenous organic matter input estimated as described by Büchi et al. (2019). ** and * indicate significant regression coefficients at $\mathbf{p}<0.01$ and $\mathbf{p}<0.05$, respectively, ns indicates nonsignificant regression coefficients. $\mathbf{R}^{2}$ represents multiple r-squared.

\begin{tabular}{ccccccc}
\hline$\Psi[\mathbf{h P a}]$ & Depth [cm] & Ka $\left[\boldsymbol{\mu \mathbf { m } ^ { 2 }}\right]$ & Clay [\%] & OC Inp [kg C ha $\left.\mathbf{~ y}^{-1}\right]$ & Int & $\mathbf{R}^{2}$ \\
\hline 30 & $-12.5 \mathrm{~cm}$ & $0.156^{*}$ & $0.872^{* *}$ & $0.005 \mathrm{~ns}$ & $-7.739 \mathrm{~ns}$ & 0.61 \\
& $-37.5 \mathrm{~cm}$ & $0.308^{* *}$ & $0.652^{* *}$ & $-0.001 \mathrm{~ns}$ & $-6.931^{*}$ & 0.77 \\
\multirow{2}{*}{100} & $-12.5 \mathrm{~cm}$ & $0.077^{*}$ & $0.933^{* *}$ & $0.005 \mathrm{~ns}$ & $-10.02 \mathrm{~ns}$ & 0.63 \\
& $-37.5 \mathrm{~cm}$ & $0.212^{* *}$ & $0.693^{* *}$ & $-0.001 \mathrm{~ns}$ & $-8.385^{*}$ & 0.73 \\
\hline
\end{tabular}

Abbreviations: $\Psi$ is soil matric suction, Ka is air permeability, Clay is clay content, OC Inp is Exogenous organic carbon input, Int is intercept

Supplemental Table S12: Summary statistics of multiple linear regression models (Eq. 3) to explain soil organic carbon content as a function of air-filled porosity, clay content and exogenous organic matter input estimated as described by Büchi et al. (2019). ** indicates significant regression coefficients at $p<0.01$, ns indicates nonsignificant regression coefficients. $R^{2}$ represents multiple $r$ squared.

\begin{tabular}{ccccccc}
\hline $\boldsymbol{\Psi}[\mathbf{h P a}]$ & Depth $[\mathbf{c m}]$ & $\boldsymbol{\varepsilon a ~}\left[\mathbf{c m}^{\mathbf{3}} \mathbf{~ c m}^{-3}\right]$ & Clay [\%] & OC Inp [kg C ha' $\left.\mathbf{y}^{-\mathbf{1}}\right]$ & Int & $\mathbf{R}^{\mathbf{2}}$ \\
\hline 30 & $-12.5 \mathrm{~cm}$ & $0.407 \mathrm{~ns}$ & $0.838^{* * *}$ & $0.006 \mathrm{~ns}$ & $-9.710 \mathrm{~ns}$ & 0.54 \\
& $-37.5 \mathrm{~cm}$ & $0.576 \mathrm{~ns}$ & $0.731^{* *}$ & $0.003 \mathrm{~ns}$ & $-13.63 \mathrm{~ns}$ & 0.48 \\
\multirow{2}{*}{100} & $-12.5 \mathrm{~cm}$ & $0.564 \mathrm{~ns}$ & $0.855^{* *}$ & $0.006 \mathrm{~ns}$ & $-13.69 \mathrm{~ns}$ & 0.57 \\
& $-37.5 \mathrm{~cm}$ & $0.607 \mathrm{~ns}$ & $0.757^{* *}$ & $0.003 \mathrm{~ns}$ & $-15.74 \mathrm{~ns}$ & 0.49
\end{tabular}

Abbreviations: $\Psi$ is soil matric suction, $\varepsilon$ a is air-filled porosity, Clay is clay content, OC Inp is Exogenous organic carbon input, Int is intercept

Supplemental Table S13: Summary statistics of multiple linear regression models (Eq. 3) to explain soil organic carbon content as a function of water holding capacity, clay content and exogenous organic matter input estimated as described by Büchi et al. (2019). ** indicates significant regression coefficients at $\mathbf{p}<0.01$, ns indicates nonsignificant regression coefficients. $\mathbf{R}^{2}$ represents multiple r-squared.

\begin{tabular}{cccccc}
\hline Depth [cm] & WHC $\left[\mathrm{g} \mathrm{g}^{-1}\right]$ & Clay [\%] & OC Inp $\left[\mathbf{k g ~ C ~ h a ~}^{-1} \mathbf{y}^{-1}\right]$ & Int & $\mathbf{R}^{\mathbf{2}}$ \\
\hline$-12.5 \mathrm{~cm}$ & $87.58^{* *}$ & $0.226 \mathrm{~ns}$ & $0.006 \mathrm{~ns}$ & $-14.95^{* *}$ & 0.67 \\
$-37.5 \mathrm{~cm}$ & $86.37^{* *}$ & $0.072 \mathrm{~ns}$ & $0.004 \mathrm{~ns}$ & $-11.95^{* *}$ & 0.60 \\
\hline
\end{tabular}

Abbreviations: WHC is water holding capacity, Clay is clay content, OC Inp is Exogenous organic carbon input, Int is intercept 

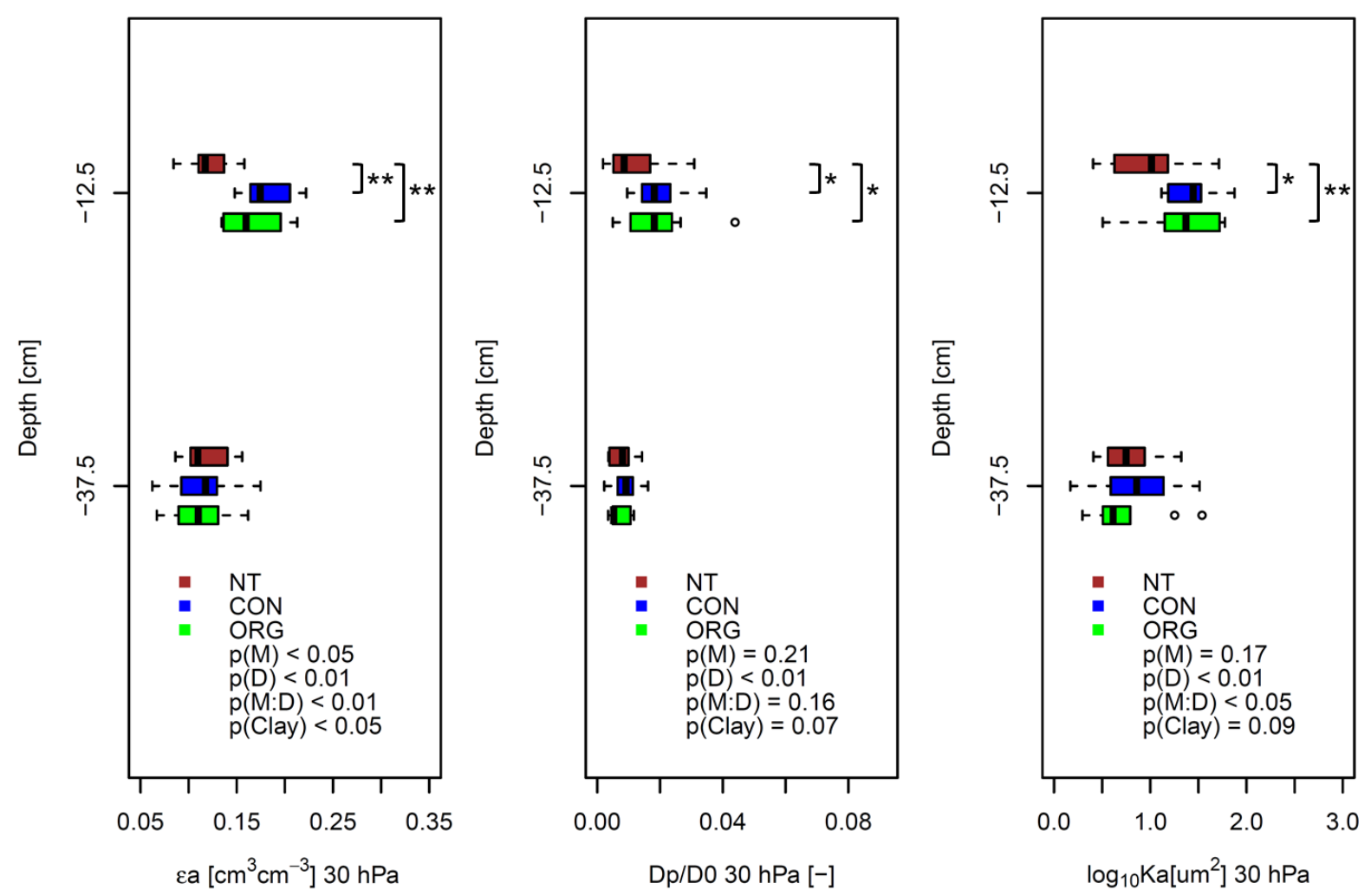

Supplemental Figure S1: Effects of soil management (M), sampling depth (D), their interaction (M:D) and clay content (Clay) on air-filled porosity, gas diffusivity and air permeability at $30 \mathrm{hPa}$ analysed with linear mixed models (Eq. 2) followed by analysis of covariance. NT (red), CON (blue) and ORG (green) denote no-till, conventional and organic management system, respectively. **

5 and * indicate significant differences between management systems at individual depths using least significant difference tests at $p$ $<0.01$ and $\mathrm{p}<0.05$, respectively $(\mathrm{n}=10)$. 

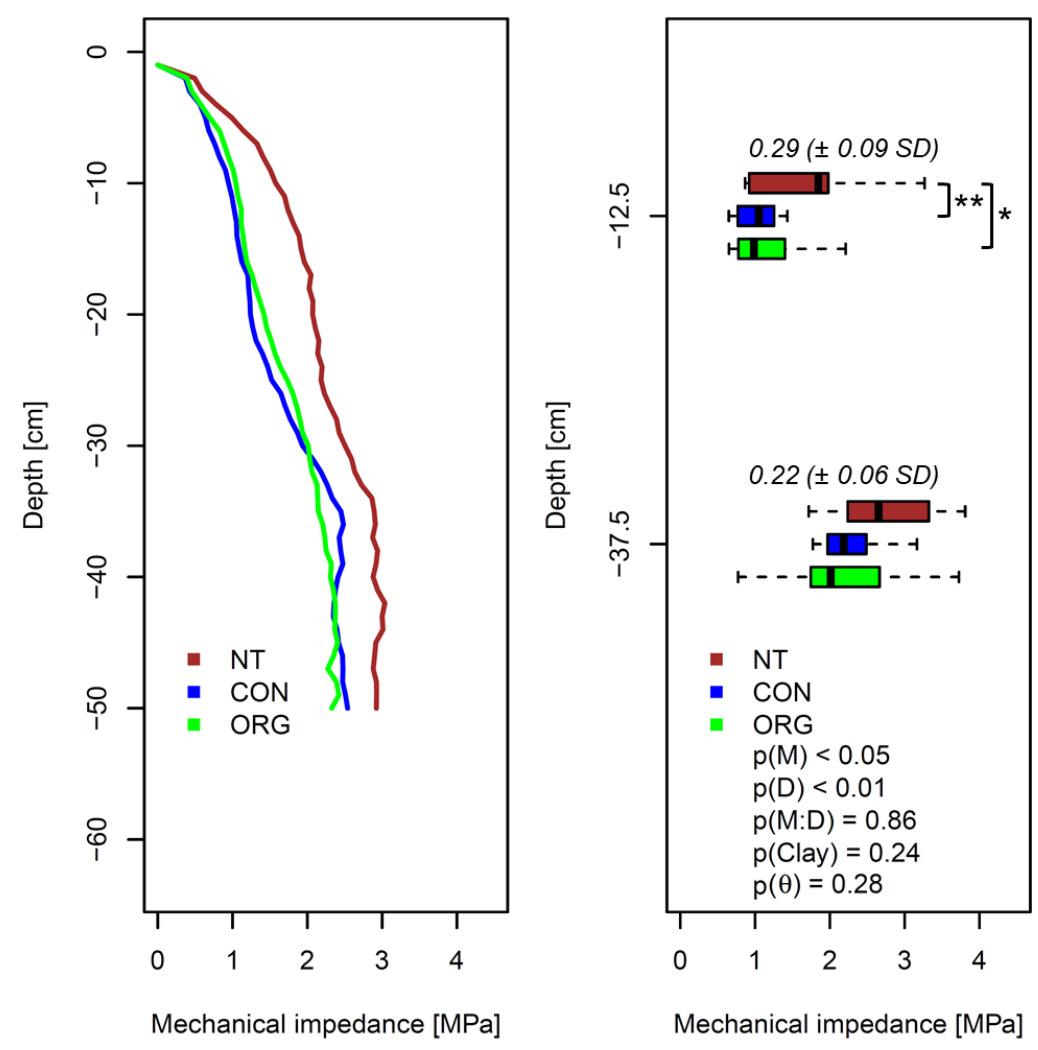

Supplemental Figure S2: Effects of soil management (M), sampling depth (D), their interaction (M:D), clay content (Clay) and gravimetric water content at sampling $(\theta)$ on soil penetration resistance analysed with linear mixed models (Eq. 2) followed by analysis of covariance. NT (red), CON (blue) and ORG (green) denote no-till, conventional and organic management system,

5 respectively. $* *$ and $*$ indicate significant differences between management systems at individual depths using least significant difference tests at $p<0.01$ and $p<0.05$, respectively $(n=10)$. Numbers in italic denote overall mean gravimetric water content $( \pm$ standard deviation) at the time of measurement. 
Gas diffusivity
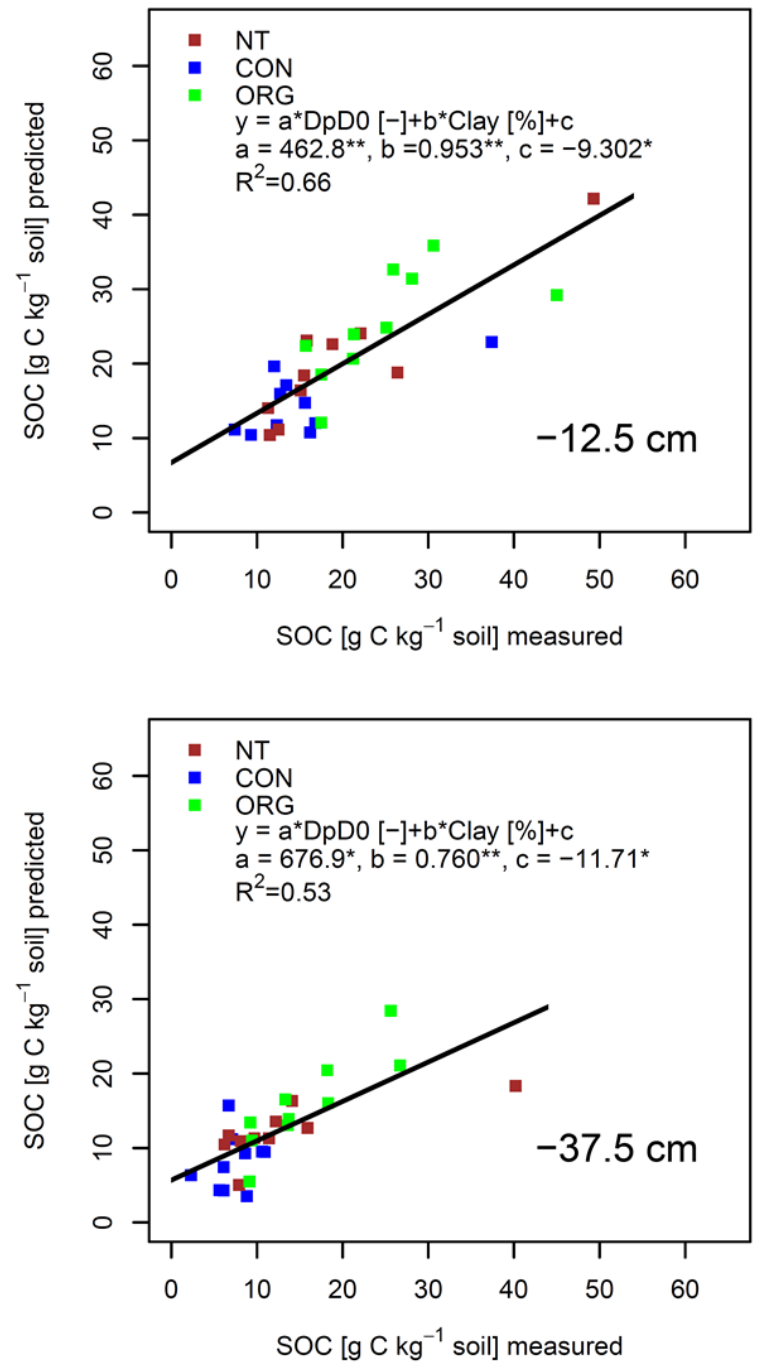

Air permeability
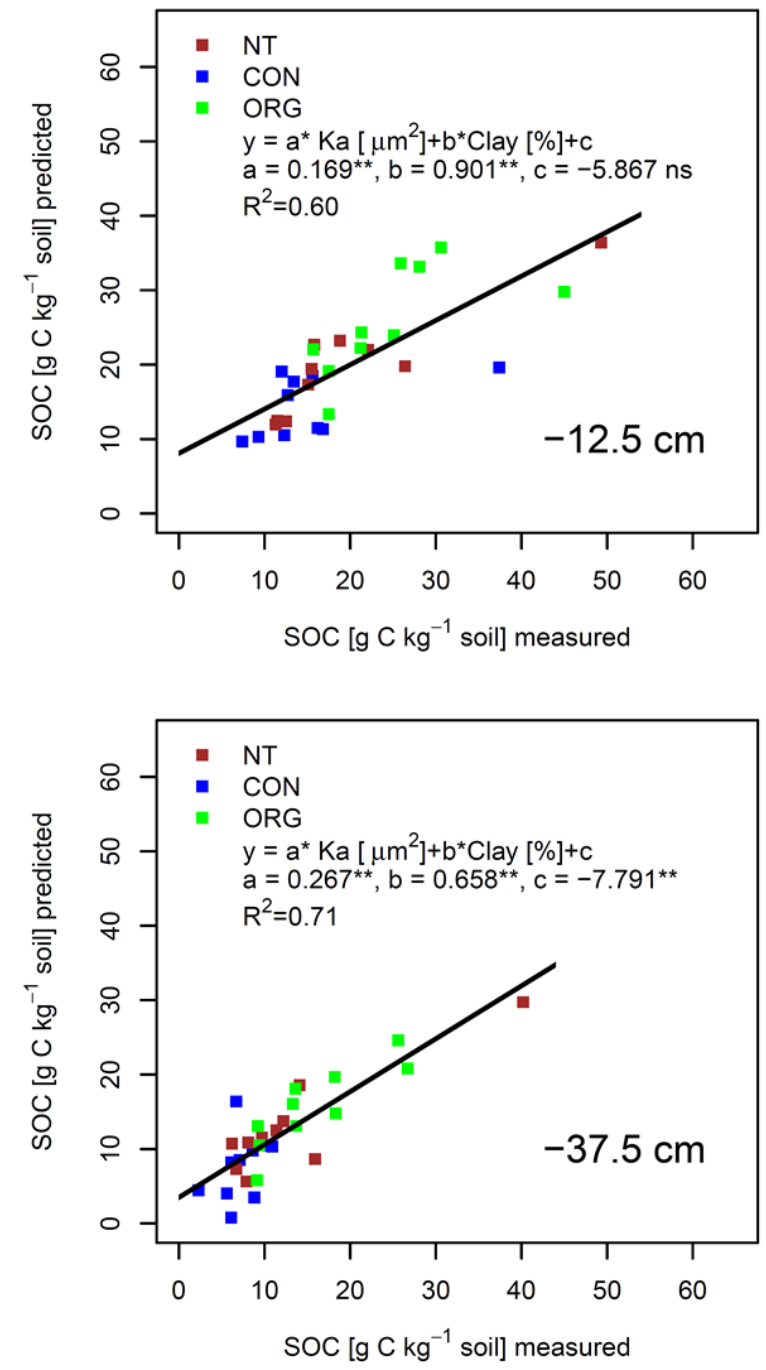

Supplemental Figure S3: Multiple linear regression models (Eq. 3) to explain soil organic carbon content as a function of gas diffusion coefficients (Dp/Do [-]) and air permeability $\left(\mathrm{Ka}^{\mathrm{C}}\left[\mathrm{\mu m}^{2}\right]\right)$ measured at $30 \mathrm{hPa}$ matric suction and clay content $(\mathrm{Clay}[\%])$. NT (red), CON (blue) and ORG (green) denote no-till, conventional and organic management system, respectively. ** and * indicate

5 significant regression coefficients at $p<0.01$ and $p<0.05$, respectively, ns indicates nonsignificant regression coefficients. $R^{2}$ represents multiple r-squared. 

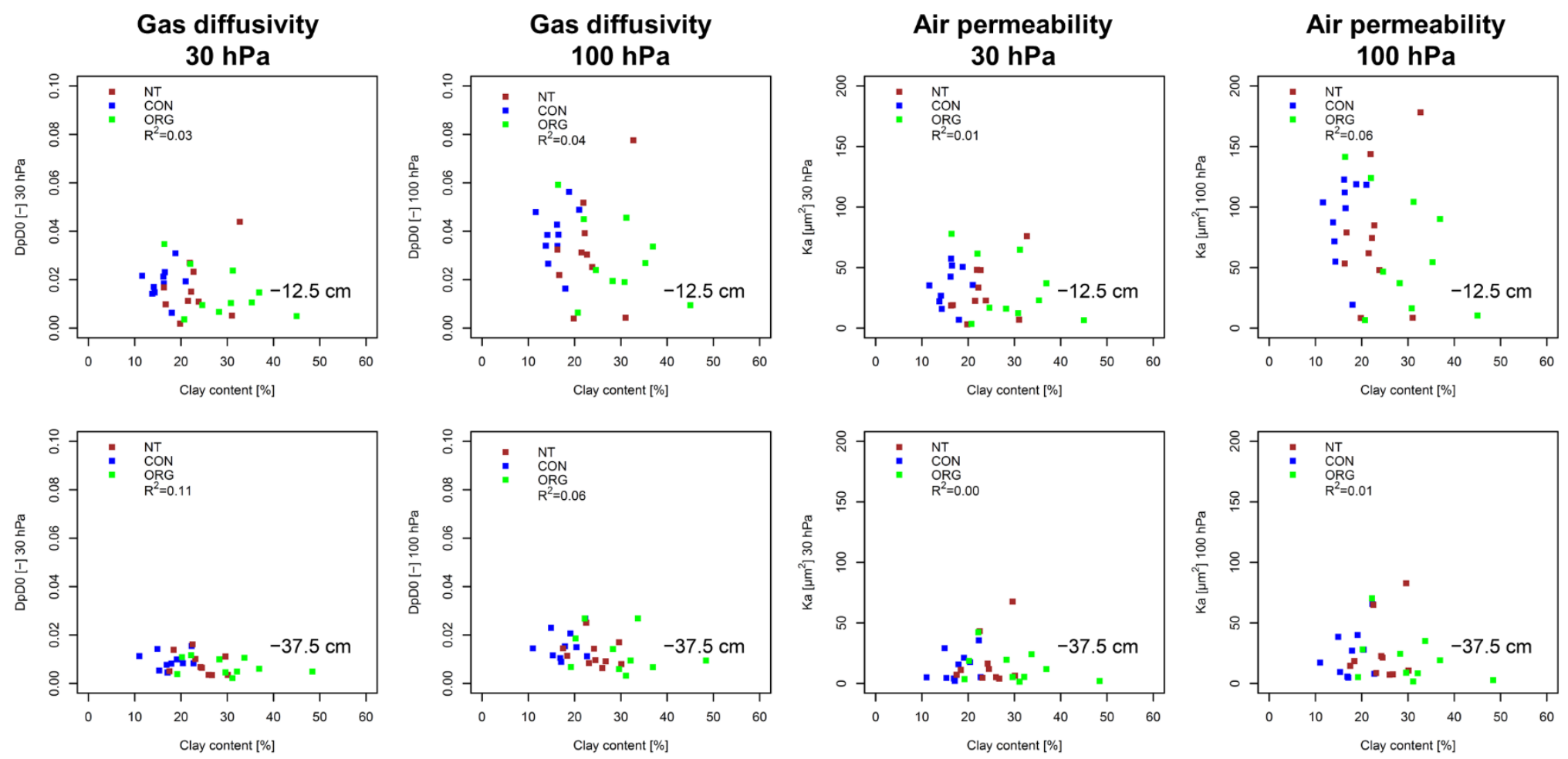

Supplemental Figure S4: Linear regressions between clay content and gas diffusion coefficients (Dp/D0 [-]) and air permeability $\left.\left(\mathrm{Ka}^{2} \mu \mathrm{m}^{2}\right]\right)$, respectively. NT (red), CON (blue) and ORG (green) denote no-till, conventional and organic management system, respectively. $R^{2}$ represents multiple r-squared. 
Air-filled porosity (30 hPa)
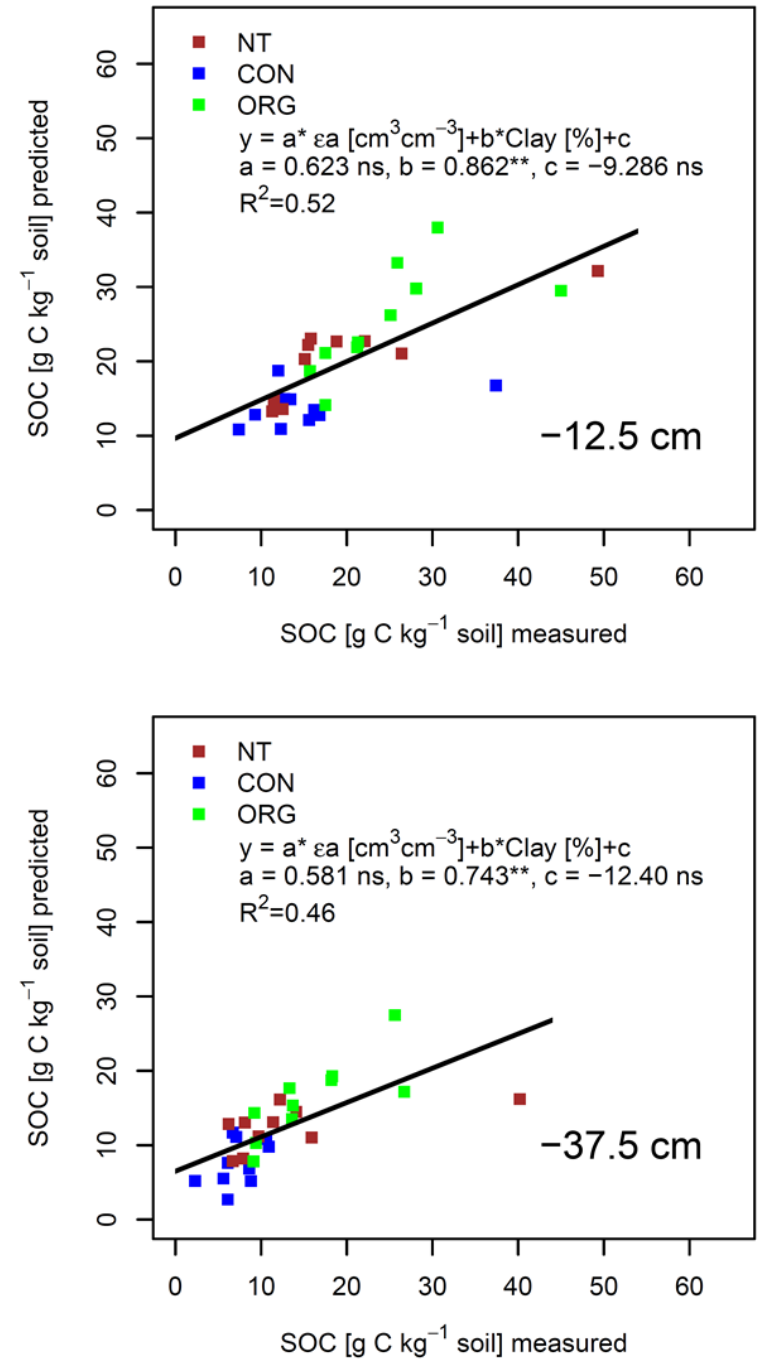

Air-filled porosity $(100 \mathrm{hPa})$
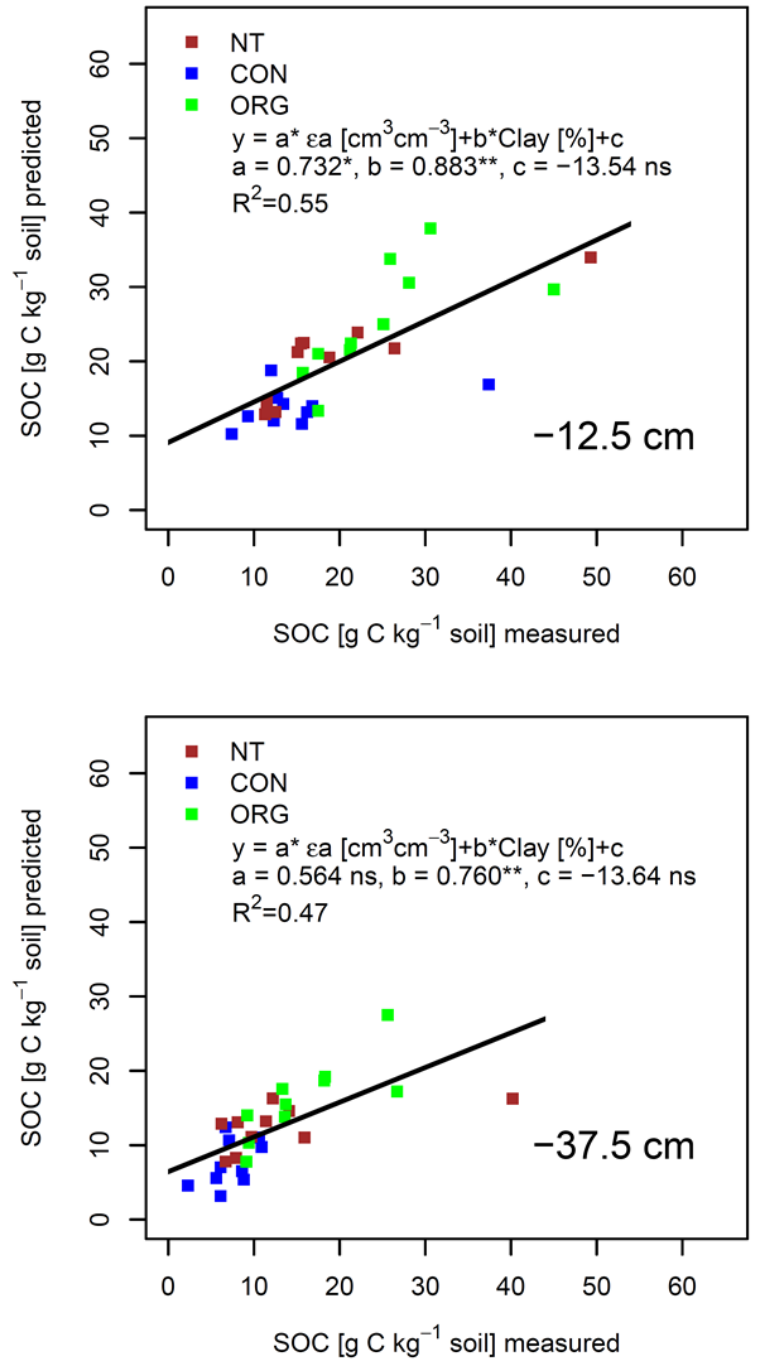

Supplemental Figure S5: Multiple linear regression models (Eq. 3) to explain soil organic carbon content as a function of air-filled porosity $\left(\varepsilon a\left[\mathrm{~cm}^{3} \mathrm{~cm}^{-3}\right]\right)$ measured at $30 \mathrm{hPa}$ and $100 \mathrm{hPa}$ matric suction and clay content (Clay [\%]). NT (red), CON (blue) and ORG (green) denote no-till, conventional and organic management system, respectively. ** and * indicate significant regression 5 coefficients at $\mathbf{p}<0.01$ and $\mathbf{p}<0.05$, respectively, ns indicates nonsignificant regression coefficients. $\mathbf{R}^{2}$ represents multiple r-squared. 
WHC $=\mathbf{f}($ Clay content $)$

$S O C=f(W H C)$
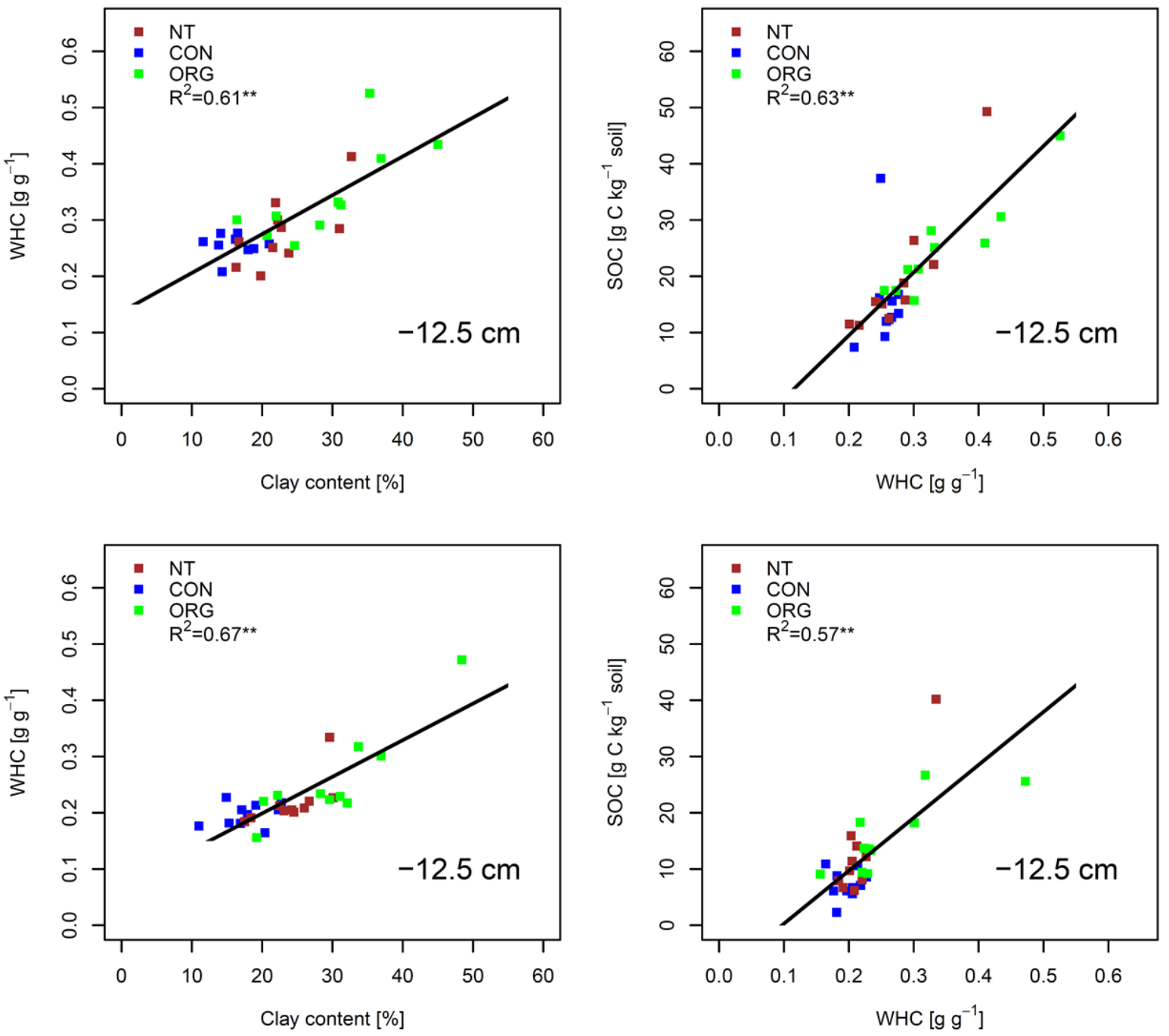

Supplemental Figure S6: Linear regressions between clay content and water holding capacity (WHC $\left.\left[\mathrm{g}^{3} \mathrm{~g}^{-3}\right]\right)$, and between water holding capacity and soil organic carbon content (SOC [g C kg-1 soil]). NT (red), CON (blue) and ORG (green) denote no-till, conventional and organic management system, respectively. $* *$ denotes significant regressions at $\mathbf{p}<0.01$ and $\mathbf{R}^{2}$ represents multiple r-squared. 